\title{
Article \\ Postural Instability after Stepping on a Stair in Older Adults: A Pilot Study
}

\author{
Hyokeun Lee ${ }^{1}$ and Kyungseok Byun ${ }^{1,2, *}$
}

\section{check for}

updates

Citation: Lee, H.; Byun, K. Postural Instability after Stepping on a Stair in Older Adults: A Pilot Study. Appl. Sci. 2021, 11, 11885. https://doi.org/ 10.3390/app112411885

Academic Editor: Nyeonju Kang

Received: 28 October 2021

Accepted: 13 December 2021

Published: 14 December 2021

Publisher's Note: MDPI stays neutral with regard to jurisdictional claims in published maps and institutional affiliations.

Copyright: (c) 2021 by the authors. Licensee MDPI, Basel, Switzerland. This article is an open access article distributed under the terms and conditions of the Creative Commons Attribution (CC BY) license (https:/ / creativecommons.org/licenses/by/ $4.0 /)$.
1 Biomechanics Research Laboratory, Vector Biomechanics, Inc., Seoul 08506, Korea; leeh82@gmail.com

2 Department of Leisure \& Sports, Kyungpook National University, Sangju 37224, Korea

* Correspondence: vbiomechanics@gmail.com; Tel.: +82-10-2876-3815

\begin{abstract}
This study aimed to examine how older adults (OA) control their postural stability after stepping on a stair in comparison to young adults (YA). Ten OA and 10 YA participated in this study. Participants ascended a single stair $(15 \mathrm{~cm}$ high by $30 \mathrm{~cm}$ wide) which was secured atop one of the force plates. Ground reaction forces (GRFs) and center of pressure (COP) motion data were obtained from the force plate under the stair. After standing on the stair with both feet, GRFs and COP data for a $3 \mathrm{~s}$ duration were analyzed to assess postural variables, including time to stabilization (TTS), COP velocity (COPVEL), and COP sway area (COPSWAY). A significant difference in TTS in the anterior-posterior direction between OA and YA $(p=0.032)$ was observed, indicating that OA had difficulty stabilizing their body posture after the stair ascent compared to YA. For COP postural variables, no significant differences in COPVEL $(p=0.455)$ and COPSWAY $(p=0.176)$ were observed between OA and YA. Study findings indicate that older adults have less capacity to regain postural stability compared to young adults following a challenging dynamic movement.
\end{abstract}

Keywords: postural stability; older adults; stepping on a stair; time to stabilization

\section{Introduction}

Postural instability in older adults (OA) leads to impaired balance control when performing activities of daily living, potentially causing an increased risk and incidence of falls and a reduction of independence and quality of life [1]. Given that the biomechanical mechanisms of postural instability in OA have been well documented [2], the balance deficits of OA have been shown to manifest not only during static movements [3], but also during dynamic transitive movement in daily activities [4].

A stair ascent task is a functionally relevant motor task that significantly challenges the locomotor and postural control system. Biomechanically, stair ascent requires significant momentum, which is necessary for conjoint upward and forward body propulsion [5]. Indeed, stabilizing one's body posture following stair ascent is needed to offset the propulsive momentum generated by the whole body. Accordingly, individuals with strength deficits may be more impaired at controlling stability after a stair ascent task, exhibiting alterations in their strength compensation strategy. Indeed, it has been reported that individuals with muscular and neurologic deficits (e.g., osteoarthritis and stroke) are at a greater risk of having a stepping-related fall due to biomechanical and environmental constraints [6,7].

When comparing OA and YA (young adults), OA are at higher risk of loss of balance and falls during stair ascent in comparison to $\mathrm{YA}$, in part due to muscular deficits. Specifically, muscle weakness in the elderly causes abnormal gait patterns and changes their gait biomechanics, particularly affecting gait velocity, and with less strength in their lower extremities leading to their greater incidence of falls $[8,9]$. Despite greater predicted possibilities that OA show balance problems and falls, studies have thus far focused less on motor control in dynamic activities, such as stepping up stairs, and even less on comparatively examining $\mathrm{OA}$ and $\mathrm{YA}$. 
Additionally, stair ascent is a complex task that is cognitively demanding compared to other simple tasks (e.g., sit-to-stand) [10], and age differences in cognitive abilities have been clearly shown [11]. A study proposed that significantly more attentional resources are required during stair ascent in OA than YA, while greater attentional resources are not required during simple tasks, such as standing [12]. It is also highlighted that cognitive decline is a common problem observed in the elderly [13]. Thus, stair ascent task assessments provide important insights into biomechanical abilities that cannot be captured through simple tasks for OA, while being sensitive to different cognitive abilities by age.

The purpose of the current study was to examine how stabilizing capabilities after stepping up stairs in OA faired in comparison to YA, hypothesizing that OA would take longer to stabilize their body posture and would have less ability to regain static plateau after the stepping performance. To find the exact time point at which one's postural sway is in plateau, we utilized the 'time to stabilization (TTS)' metric, which provides underlying information calculated based on overall information in a time series. It is useful and applicable when assessing dynamic balance capability.

\section{Materials and Methods}

\subsection{Participants}

Ten OA (Age: $71 \pm 4.2 \mathrm{yr}$, height: $170.6 \pm 5.4 \mathrm{~cm}$, body mass: $73.2 \pm 9.4 \mathrm{~kg}$ ) and 10 YA (age: $27 \pm 6 \mathrm{yr}$, height: $172.1 \pm 7.4 \mathrm{~cm}$, body mass: $72.5 \pm 13.8 \mathrm{~kg}$ ) participated in this study. All participants were asked to perform a cognition test (the mini-mental state examination, or MMSE) for screening purposes, and those who obtained a score of 23 or under were excluded from participating in the study. Participants had not had any musculoskeletal problems within the past six months and had not had any recent surgery. Informed consent was reviewed with each participant, and once all questions were answered and the documentation of consent was obtained, the experimental session began.

\subsection{Experimental Protocol}

Sixteen passive reflective markers were attached to the lower body in accordance with the instructions that accompanied the Helen Hays marker set, and kinematic data were collected using a 10-camera motion capture system $(100 \mathrm{~Hz}$, Qualisys, Gothenburg, Sweden). For the stair ascent trial, participants ascended a single stair $(18 \mathrm{~cm}$ high by $40 \mathrm{~cm}$ wide), which was secured atop one of the force plates, barefoot. In response to a verbal signal of "ready", participants were asked to wait a moment and then begin the movement by stepping onto the stair with their dominant leg and maintain their stability (for at least $5 \mathrm{~s}$ ) in a static position once both feet were atop the stair. Further, during the stepping task, participants were asked to fold their arms across their chest. The events of the stepping performance, including (1) the initial foot being raised from the ground, (2) the initial foot making contact with the stair, (3) the second foot being raised from the ground, and (4) the second foot making contact with the stair, were identified based on the ground reaction force data and the feet kinematic data. Ground reaction forces (GRFs) and moments were recorded using two force plates, one mounted on the laboratory floor and the other on the stair $(300 \mathrm{~Hz}$, Kistler, Winterthur, Swiss) (Figure 1). We measured GRFs and center of pressure (COP) motion along the anterior-posterior (AP) and mediolateral (ML) axes of motion for data analysis, and filtered GRF and COP data using a second-order Butterworth low-pass filter with a cutoff frequency of $5 \mathrm{~Hz}$. GRFs and COP data were captured for a duration of $3 \mathrm{~s}$ after both feet were standing on the stair to assess the subjects' postural stabilization. 


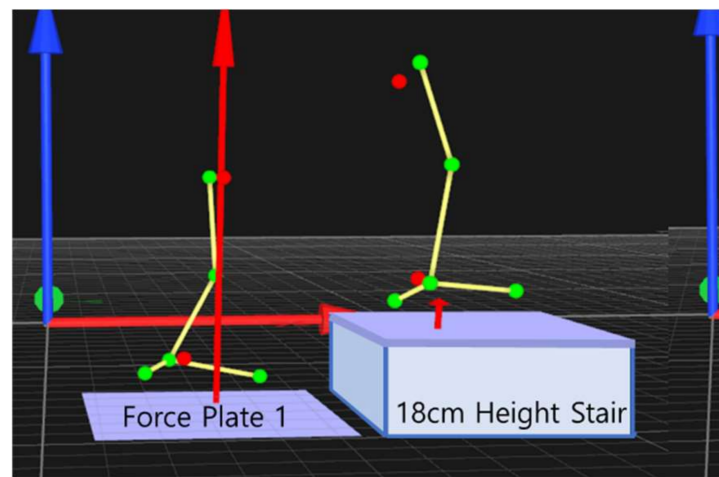

Initial foot contact onto the stair

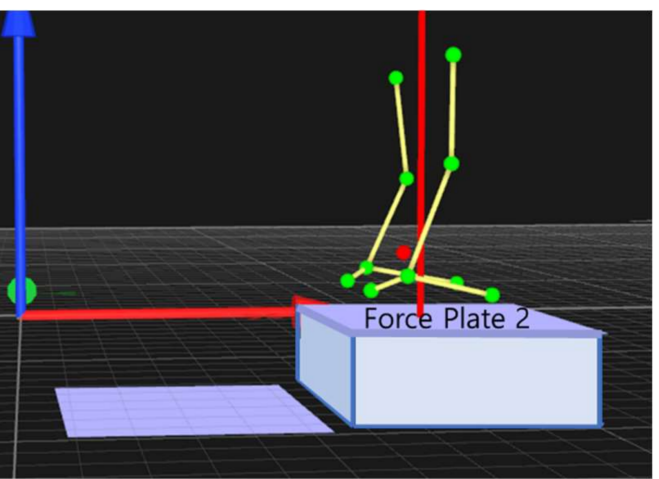

Second foot contact onto the stair

Figure 1. Experimental protocol of the stepping on a stair task with two force plates, one mounted on the laboratory floor (the first plate) and the other on the stair (the second plate).

\subsection{Data Reduction and Processing}

We obtained GRF and center of pressure (COP) motion data along the anteriorposterior (AP) and medial-lateral (ML) axes of motion for data analysis, and filtered the GRF and COP data using a second-order Butterworth low-pass filter with a cutoff frequency of $5 \mathrm{~Hz}$. GRFs and COP data were measured for a duration of $3 \mathrm{~s}$ after both feet were standing on the stair (event four: when the second foot came into contact with the stair) to assess the subjects' postural stabilization.

Time to stabilization (TTS) scores for the AP and ML directions were separately calculated according to the ground reaction forces ( $\mathrm{x}$ vector, AP; y vector, $\mathrm{ML}$ ). As a sequential estimation, TTS incorporates an algorithm to calculate a cumulative average of the data points in a series by successively adding one point at a time [14]. This cumulative average value is sequentially compared with the overall series mean. When the value of the sequential average passes through a level that is within $0.25 \mathrm{SDs}$ of the overall series mean, the individual series is considered to be at a plateau stage. The series consists of all data points within the first $3 \mathrm{~s}$ of both feet making contact with the stair (Figure 2).
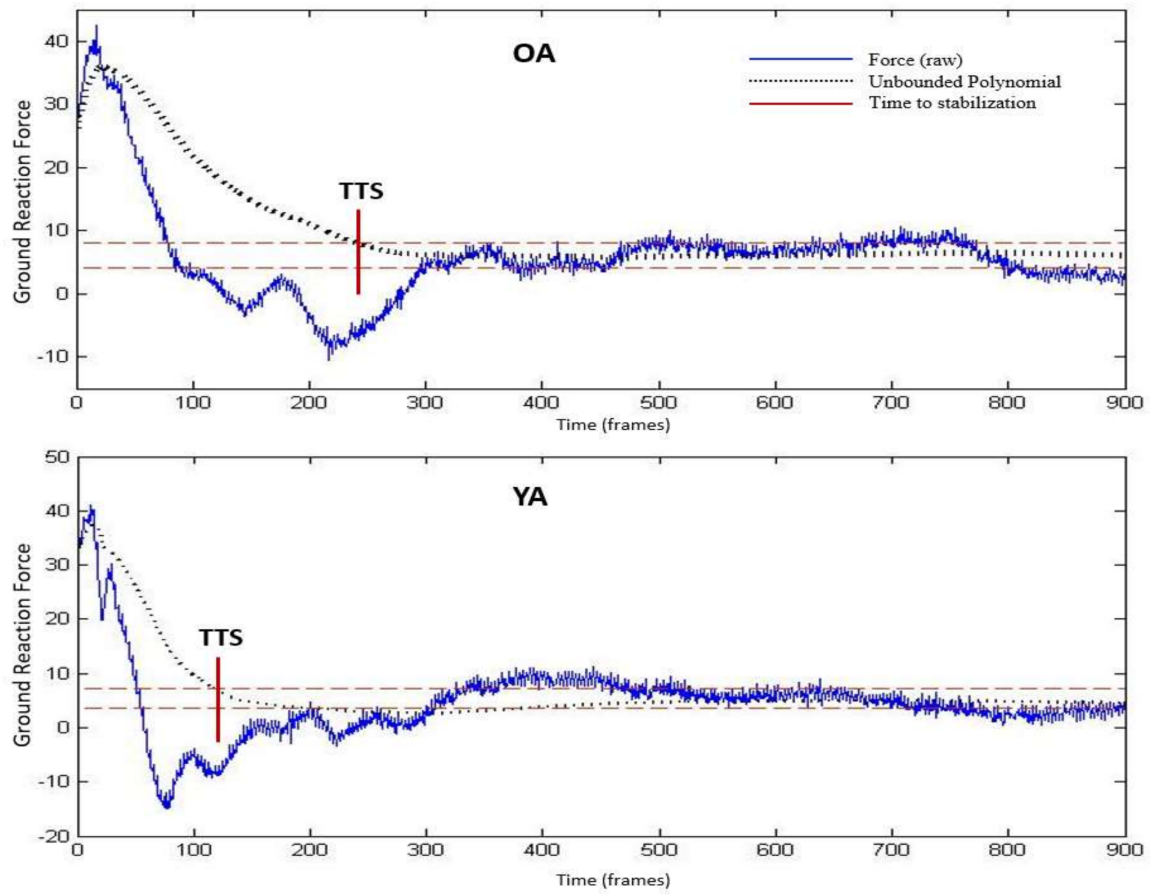

Figure 2. Time to stabilization in OA and YA calculated using ground reaction forces measure-ments. 
For the traditional postural assessment, we also calculated COP velocity (COPVEL) and sway of $95 \%$ confidence ellipse (COPSWAY), which were calculated around the filtered COP motion along both the AP and ML axes (Figure 3). The details regarding the procedure to calculate COPVEL and the COPSWAY are described in previous literature $[15,16]$.
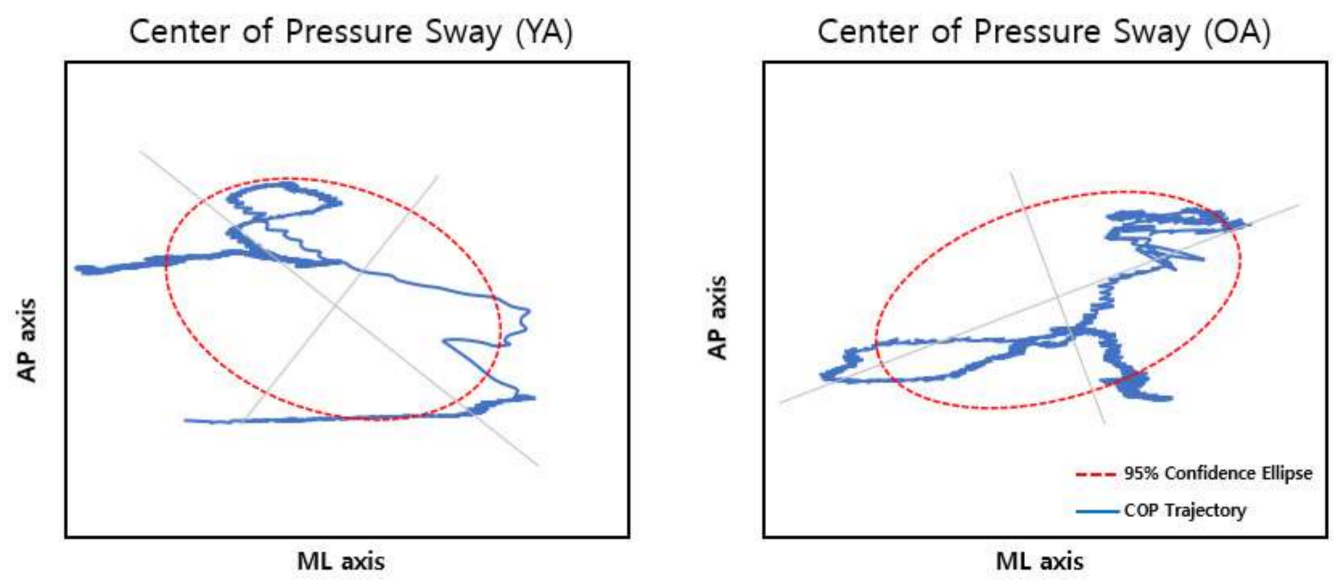

Figure 3. The center of pressure (COP) trajectories during posture stabilization after a stepping movement in OA and YA.

\subsection{Statistical Analysis}

Descriptive statistics for age, body mass, height, and MMSE were calculated for both groups. Independent $t$-tests were used to compare all dependent variables between $\mathrm{OA}$ and YA. Statistical analyses were performed using SPSS, and all levels of significance were set at $\alpha=0.05$.

\section{Results}

A significant difference in TTS AP between OA and YA $(p=0.032)$ and a marginal but not statistical difference in TTS ML $(p=0.141)$ were observed, indicating that OA needed a longer time to stabilize their body posture after stair ascent compared to YA. For COP postural variables, no significant difference in COPVEL $(p=0.455)$ and COPSWAY $(p=0.176)$ were observed between OA and YA (Table 1).

Table 1. Mean, standard deviation, and $p$-value from $t$-tests for all dependent variables, including TTS AP, TTS ML, COPVEL, and COPSWAY. $p<0.05$ for difference between OA and YA.

\begin{tabular}{cccc}
\hline Variables & Older Adults (OA) & Young Adults (YA) & $p$-Value \\
\hline Time to Stabilization (AP) & $1.62 \pm 0.15$ & $1.26 \pm 0.12$ & $p=0.032^{*}$ \\
Time to Stabilization (ML) & $1.85 \pm 0.21$ & $1.82 \pm 0.23$ & $p=0.141$ \\
COPVEL (cm/s) & $0.84 \pm 0.23$ & $0.81 \pm 0.25$ & $p=0.455$ \\
COPSWAY (CE95\%, $\mathrm{cm}^{2}$ ) & $31.4 \pm 12.1$ & $0.81 \pm 0.25$ & $p=0.176$ \\
\hline
\end{tabular}

* Significant difference between OA and YA at $p<0.05$.

\section{Discussion}

There have been only a few studies using time to stabilization (TTS) to assess postural capacity in older adults (OA) or disease populations. A previous study utilized TTS to assess how stroke patients control their postural stability in response to unpredicted perturbation [17]. They reported that stroke patients who had intensive weighted training showed decreased TTS scores, indicating their improved capacity to stabilize their postural sway in the face of unpredicted perturbations. Bieryla and Madigan [18] further demonstrated that an improvement in one's postural stability as measured by TTS was observed more in older adults who had exercise training than those without training.

To our knowledge, this preliminary investigation is the first to assess the stabilization capacity of OA following a stepping movement, as measured by TTS. There have been a 
few studies that have compared spatiotemporal measures and the stabilizing strategies of OA and YA during stair ascent. One study reported that there were no significant group differences in stepping performance [19], while another study reported that OA show smaller separations between the center of mass and the center of pressure, which is indicative of different stepping strategies between groups [20]. However, no study examined the stabilization capacity after the stepping movement had been completed. By using TTS in this study, we tried to account for the deficits of stabilizing capacity in OA during a challenging dynamic movement. As hypothesized, OA exhibited a significantly longer time to stabilize their postural sway following stair ascent in comparison to YA. This finding indicated that OA may have less capacity to regain postural stability compared to YA following a challenging task, such as stepping on a stair. Given that a longer time for stabilization is highly correlated with a greater risk of falls [21], our TTS finding supports the observation that OA are at a greater risk of falls compared to YA. The finding of the current study could potentially provide a reliable and objective index for the evaluation of dynamic postural stability in OA.

However, we did not observe any significant differences between OA and YA in balance performance during posture stabilization after stepping on a stair when we assessed this using COP measures (e.g., COPVEL and COPSWAY). Conventionally, balance problems in OA have been identified by evaluating the COP motion [22]. For example, greater velocity or sway area during static movement has been considered to be one of the representative characteristics of postural control deficits in OA [23]. Biomechanically, however, dynamic movement, such as stair ascent, require a sufficient level of body momentum and co-contraction between COP and the body's center of mass to maintain postural stability when compensating for propulsive body momentum [24]. Unlike static standing, such dynamic movements are likely performed with greater variation because the majority of factors (e.g., force generation, cognition, and the environment itself) are consistently working as determining contributors to overall dynamic performance [25]. Therefore, it is difficult to account for postural capacity by using traditional COP measures, such as sway area or velocity, when biomechanically investigating dynamic balance.

There were limitations to the current pilot study. In a previous biomechanical study measuring TTS in athlete populations, it was reported that ankle joint stability, braces, and fatigue were closely related to TTS, indicating that ankle joint functions play a crucial role in dynamic balance and time to stabilization when experiencing an external perturbation [26]. Although previous studies investigated the balance recovery function using the TTS measure, which is consistent with our investigation, results from the current study should be interpreted with careful consideration. Particularly, unlike a previous study [27], subjects in our investigation were screened out when they had any musculoskeletal problems and fatigue. Therefore, we speculate that there might be other factors that affect TTS scores (e.g., muscle strength), which clearly differentiated the balance recovery functions between $\mathrm{OA}$ and YA.

\section{Conclusions}

The findings in this study will have the potential to give us a better understanding of how the elderly experience postural instability in daily life. More research is needed, however, to confirm the current findings and expand our understanding of what constitutes meaningful biomechanical change according to TTS scores in OA. The capability to stabilize one's posture after completing a dynamic movement is primarily based on how a person negotiates various physical constraints that result from neuroanatomical, biomechanical, and environmental origins. Thus, to better understand the neuromuscular system underlying postural control recovery strategies in OA, diverse factors, such as muscle power and psychiatric aspects (e.g., fear of falling), on postural control should be comprehensively considered in future studies. 
Author Contributions: Conceptualization, H.L.; methodology, H.L.; writing-original draft preparation, H.L.; writing-review and editing, H.L. and K.B.; supervision, K.B.; project administration, H.L. and K.B. All authors have read and agreed to the published version of the manuscript.

Funding: This research received no external funding.

Institutional Review Board Statement: The study was conducted according to the guidelines of the Declaration of Helsinki, and approved by the Institutional Review Board of the University of Florida (IRB201401029).

Informed Consent Statement: Informed consent was obtained from all participants involved in the study.

Data Availability Statement: Not applicable.

Acknowledgments: We thank the study participants for their time and effort.

Conflicts of Interest: The authors declare no conflict of interest.

\section{References}

1. Murphy, S.L.; Williams, C.S.; Gill, T.M. Characteristics associated with fear of falling and activity restriction in community-Living older persons. J. Am. Geriatr. Soc. 2002, 50, 516-520. [CrossRef] [PubMed]

2. Maki, B.E.; McIlroy, W.E. Postural control in the older adult. Clin. Geriatr. Med. 1996, 12, 635-658. [CrossRef]

3. Oba, N.; Sasagawa, S.; Yamamoto, A.; Nakazawa, K. Difference in postural control during quiet standing between young children and adults: Assessment with center of mass acceleration. PLoS ONE 2015, 10, e0140235. [CrossRef]

4. Park, Y.S.; Kim, E.H.; Kim, T.W.; Lee, Y.S.; Lim, Y.T. The effects of 12-week balance ability improvement exercise to the changes of selected joint angles and ground reaction forces during down staircase walking. Korean J. Sport Biomech. 2010, 20, 267-275. [CrossRef]

5. Eun, S.D. An investigation of the effect of the height of wteps on the joint moment of lower extremities of the elderly while walking downstairs. Korean J. Sport Biomech. 2006, 16, 31-38.

6. Novak, A.C.; Brouwer, B. Kinematic and kinetic evaluation of the stance phase of stair ambulation in persons with stroke and healthy adults: A pilot study. J. Appl. Biomech. 2013, 29, 443-452. [CrossRef]

7. Startzell, J.K.; Owens, D.A.; Mulfinger, L.M.; Cavanagh, P.R. Stair negotiation in older people: A review. J. Am. Geriatr Soc. 2000, 48, 567-580. [CrossRef] [PubMed]

8. Granacher, U.; Muehlbauer, T.; Gruber, M. A qualitative review of balance and strength performance in healthy older adults: Impact for testing and training. J. Aging Res. 2012, 2012, 708905. [CrossRef] [PubMed]

9. Tinetti, M.E.; Speechley, M.; Ginter, S.F. Risk factors for falls among elderly persons living in the community. N. Engl. J. Med. 1988, 319, 1701-1707. [CrossRef]

10. Miyasike-daSilva, V.; Mcllroy, W.E. Does it really matter where you look when walking on stairs? Insights from a dual-task study. PLoS ONE 2012, 7, e44722. [CrossRef]

11. Shin, S.; Jang, D.G.; Jang, J.K.; Park, S.H. The effect of age and dual task to human postural control. Korean J. Sport Biomech. 2013, 23, 169-177. [CrossRef]

12. Ojha, H.A.; Kern, R.W.; Lin, C.H.J.; Winstein, C.J. Age affects the attentional demands of stair ambulation: Evidence from a dual-task approach. Phys. Ther. 2009, 89, 1080-1088. [CrossRef] [PubMed]

13. Parihar, R.; Mahoney, J.R.; Verghese, J. Relationship of gait and cognition in the elderly. Curr. Transl. Geriatr. Exp. Gerontol. Rep. 2013, 2, 167-173. [CrossRef]

14. Ross, S.E.; Guskiewicz, K.M. Time to Stabilization: A Method for Analyzing Dynamic Postural Stability. Int. J. Athl. Ther. 2003, 8, 37-39.

15. Colby, S.; Hintermeister, R.A.; Torry, M.R.; Steadman, J.R. Lower limb stability with ACL impairment. J. Orthop. Sports. Phys. Ther. 1999, 29, 444-451. [CrossRef]

16. Prieto, T.E.; Myklebust, J.B.; Hoffmann, R.G.; Lovett, E.G.; Myklebust, B.M. Measures of postural steadiness: Differences between healthy young and elderly adults. IEEE. Trans. Biomed. Eng. 1996, 43, 956-966. [CrossRef] [PubMed]

17. Yamamoto, T.; Smith, C.E.; Suzuki, Y.; Kiyono, K.; Tanahashi, T.; Sakoda, S.; Morasso, P.; Nomura, T. Universal and individual characteristics of postural sway during quiet standing in healthy young adults. Physiol. Rep. 2015, 3, e12329.f. [CrossRef]

18. Vearrier, L.A.; Langan, J.; Shumway-Cook, A.; Woollacott, M. An intensive massed practice approach to retraining balance post-stroke. Gait Posture 2005, 22, 154-163. [CrossRef]

19. Bieryla, K.A.; Madigan, M.L. Proof of concept for perturbation-based balance training in older adults at a high risk for falls. Arch. Phys. M. Arch. Phys. Med. 2011, 92, 841-843. [CrossRef] [PubMed]

20. Lee, H.J.; Chou, L.S. Balance control during stair negotiation in older adults. J. Biomech. 2007, 40, 2530-2536. [CrossRef]

21. Reevesa, N.D.; Spanjaardab, M.; Mohagheghia, A.A.; Baltzopoulosa, V.; Maganarisa, C.N. Older adults employ alternative strategies to operate within their maximum capabilities when ascending stairs. J. Electromyogr. Kinesiol. 2009, 19, e57-e68. [CrossRef] 
22. Shumway-Cook, A.; Baldwin, M.; Polisssar, N.L.; Gruber, W. Predicting the probability for falls in community-dwelling older adults. Phys Ther. 1997, 77, 812-819. [CrossRef]

23. Roman-Liu, D. Age-related changes in the range and velocity of postural sway. Arch. Gerontol. Geriatr. 2018, 77, 66-80. [CrossRef] [PubMed]

24. Lin, D.; Seol, H.; Nussbaum, M.A.; Madigan, M.L. Reliability of COP_based postural sway measures and age-related differences. Gait Posture 2008, 28, 337-342. [CrossRef] [PubMed]

25. Costigan, P.A.; Deluzio, K.J.; Wyss, U.P. Knee and hip kinetics during normal stair climbing. Gait Posture 2002, 16, 31-37. [CrossRef]

26. Della Croce, U.; Riley, P.O.; Lelas, J.L.; Kerrigan, D.C. A refined view of the determinants of gait. Gait Posture 2001, 14, 79-84. [CrossRef]

27. Shaw, M.Y.; Gribble, P.A.; Frye, J.L. Ankle bracing, fatigue, and time to stabilization in collegiate volleyball athletes. J. Athl. Train. 2008, 43, 164-171. [CrossRef] [PubMed] 